\title{
Time to absorption in discounted reinforcement models
}

\author{
Robin Pemantle ${ }^{1,2}$ \\ Brian Skyrms 3
}

\begin{abstract}
:
Reinforcement schemes are a class of non-Markovian stochastic processes. Their non-Markovian nature allows them to model some kind of memory of the past. One subclass of such models are those in which the past is exponentially discounted or forgotten. Often, models in this subclass have the property of becoming trapped with probability 1 in some degenerate state. While previous work has concentrated on such limit results, we concentrate here on a contrary effect, namely that the time to become trapped may increase exponentially in $1 / x$ as the discount rate, $1-x$, approaches 1 . As a result, the time to become trapped may easily exceed the lifetime of the simulation or of the physical data being modeled. In such a case, the quasi-stationary behavior is more germane. We apply our results to a model of social network formation based on ternary (three-person) interactions with uniform positive reinforcement.
\end{abstract}

Keywords: network, social network, urn model, Friedman urn, stochastic approximation, metastable, trap, three-player game, potential well, exponential time, quasi-stationary.

Subject classification: Primary: 60J20

\footnotetext{
${ }^{1}$ Research supported in part by National Science Foundation grant \# DMS 0103635

${ }^{2}$ Department of Mathematics, The Ohio State University, 231 W. 18 Ave., Columbus, OH 43210

${ }^{3}$ School of Social Sciences, University of California at Irvine, Irvine, CA 92607
} 


\section{Introduction}

Stochastic models incorporating mechanisms by which likelihoods of outcomes increase according to their accumulated frequencies have been around since the introduction of Pólya's Urn [EP23]. The mathematical framework for many of these models appears in the literature on stochastic approximation, beginning with [RM51], in the urn model literature [Fr65], in the literature on reinforced random walks [Pem90, Dav90, Lim01], and in the literature on the relation between stochastic systems and their deterministic mean-field dynamical system approximations [BH95, Ben96].

These processes, known in the mathematical community as reinforcement processes, have long been used by psychologists as models for learning [BM55, IT69, Nor72, Lak81]. Increasingly, reinforcement models have been adopted by other social scientists as interactive models in which collective learning takes place in the form of network formation or adaptation of strategies: sociologists studying the "small world" network phenomenon [WS98, BW00], formation of dyads of reciprocal approval [FM96]; economists studying evolutionary game theory [MS82], strategic learning [RE95] [FK93] or its interaction with network structure [Ell93, AI97]. These models are designed to explore mechanisms by which agents with limited information, rationality or sophistication may nevertheless achieve advantageous social structures via the application of simple rules for behavior change.

Due, perhaps, to a dearth of types of simple local rules (or perhaps to a lack of imagination on the part of modelers), most reinforcement models fall into one of two classes. The first class contains models for which the past is weighted uniformly. This class includes the urn models, stochastic approximations and reinforced random walks mentioned above, as well as the economic game theory models of Erev and Roth [RE95]. Uniform weighting means that the step from time $n-1$ to $n$ represents a fraction of only $1 / n$ of the total learning up to time $n$, so one obtains a time-inhomogenous process in which the hidden variables change by amounts on the order of $1 / n$ at time $n$. The second class consists of models in which the past is exponentially discounted or forgotten. This class includes the learning models of the 1960's and 70's [IT69], as well as many contemporary models of repeated economic games, e.g. [BL02]. In these models, the weight of the present is asymptotically equal to the discount parameter, $x$, defined as the $x$ for which an action $t$ units of time will be weighted by $(1-x)^{t}$. More precisely, the fraction of total learning between time $n-1$ and $n$ of the total learning to time $n$ will be roughly the maximum of $1 / n$ and $x$.

Our chief concern in this paper is to study how the discounted process approaches the nondiscounted process as $x \rightarrow 0$. The long-run behavior in these two cases is qualitatively different. Limit theorems for non-discounted processes have been obtained chiefly in the framework of stochastic perturbations to dynamical systems. Typically, the stochastic system converges to limit points or limit cycles of the dynamical system that corresponds to the mean motion of the stochastic system [BH95]. The random limit is supported on weakly stable equilibria [Pem90], though the system may remain near unstable equilibria for long periods of time (see [BP03] for a discussion of this phenomenon in continuous time, and [PS03b] for a case study in discrete time).

In the discounted processes we study here, there are trapping states, into which the chain must eventually fall (there is another kind of discounted process we are not concerned with here, which converges to an ergodic Markov chain, see, e.g., [IT69]). The reciprocity model of [BL02], for example, is of this type. From the point of view of studying the transition as discounting goes to zero, the most interesting case is when the trapping states are disjoint from the stable equilibria in 
the non-discounted process. Trapping states for the discounted process must always be equilibria for the non-discounted process, but when all the trapping states are unstable equilibria (equivalently, all the stable equilibria of the non-discounted process are non-trapping in the discounted process), then the conflict between the discounted and non-discounted behavior is maximized.

The transition is easy to describe informally. As the discount rate approaches zero, the discounted process behaves for a longer and longer time like the non-discounted process, and then abruptly falls into a trap. Of course when the discounting parameter is $x$ it takes time at least on the order of $1 / x$ for the system to notice there is discounting going on. But in fact, due to the learning that has gone on during this phase, it will take time of order $\exp \left(c x^{-1}\right)$ before the system discovers a trap and falls in. It is in fact not hard to guess this via back-of-the-napkin computations. One of our main motivations for pursuing this rigorously was to explain why simulation data contradicted the easily proved limit theorem: it was because the time scale of the simulation (let alone of any real phenomenon modeled by the simulation) was never anywhere near the time needed to find a trap.

Our purpose in the present paper is to prove various versions of this. In the next section, we present the ternary interaction model which was our original motivation for this study. Section 3 then introduces a simple process that is a building block for the ternary interaction model. For that process, results about trapping times can be proved with the correct constant. The last section then proves a $\exp \left(c x^{-1}\right)$ waiting time result for a general class of models, but without the correct value of c. With a little linear algebra, this is shown to apply to the ternary interaction model of Section 2 .

\section{Three's Company: a ternary interaction model}

The following process is described in [PS03a], where it is called Three's Company, and is put forth as a model for formation of ternary collaborations in a three-player version of Rousseau's stag hunting game. Fix a positive integer $N \geq 4$, representing the size of the population. For $t \geq 0$ and $1 \leq i, j \leq N$, define random variables $W(i, j, t)$ and $U(i, t)$ inductively on a common probability space $(\Omega, \mathcal{F}, \mathbb{P})$ as follows. The $W$ variables are positive numbers, and the $U$ variables are subsets of the population of cardinality 3 . One may think of the $U$ variables as random triangles in the complete graph with a vertex representing each agent. The initialization is $W(i, j, 0)=1$ for all $i \neq j$, while $W(i, i, 0)=0$ ). The inductive step, for $t \geq 0$, defines probabilities (formally, conditional probabilities given the past) for the variables $U(i, t)$ in terms of the variables $W(r, s, t), r, s \leq N$, and then defines $W(i, j, t+1)$ in terms of $W(i, j, t)$ and the variables $U(r, t), r \leq N$. The equations are:

$$
\begin{aligned}
\mathbb{P}\left(U(i, t)=S \mid \mathcal{F}_{t}\right) & =\frac{\mathbf{1}_{i \in S} \prod_{r, s \in S, r<s} W(r, s, t)}{\sum_{S^{\prime}: i \in S^{\prime}} \prod_{r, s \in S^{\prime}, r<s} W(r, s, t)} \\
W(i, j, t+1) & =(1-x) W(i, j, t)+\sum_{r=1}^{N} \mathbf{1}_{i, j \in U(r, t)}
\end{aligned}
$$

Here $(1-x)$ is the factor per unit time by which the past is discounted, and the $\sigma$-field conditioned on is the process up to time $t$,

$$
\mathcal{F}_{t}:=\sigma\{W(i, j, u): u \leq t\}
$$


We may think of the normalized matrix

$$
\mathbf{W}_{t}:=\frac{1}{\sum_{i, j} W(i, j, t)} W(\cdot, \cdot, t)
$$

as the state vector, which is then an asymptotically time-homogeneous Markov chain, with an evolution rule of the well known form

$$
\mathbb{E}\left(\mathbf{W}_{t+1}-\mathbf{W}_{t} \mid \mathcal{F}_{t}\right)=g(t)\left[\mu\left(\mathbf{W}_{t}\right)+\xi_{t}\right]
$$

where in this case, $g(t)=1 / x+O(1 / t)$, the drift vector field $\mu$ maps the simplex of normalized matrices into its tangent space and may be explicitly computed, and $\xi_{t}$ are martingale increments of order 1.

These equations model a social interaction in which each agent $i$ at each time $t$ invites two others to frolic ${ }^{4}$. For each agent $i$, the trio chosen by $i$ is chosen from all possible trios containing $i$, according to the products of the weights $W(i, \cdot, t)$. Thus the probability of agent $i$ forming the trio $\{i, j, k\}$ is proportional to $W(i, j, t) W(i, k, t)$. After the frolicking, fond memories ensue: each of the three pair weights $W(i, j, t), W(i, k, t)$ and $W(j, k, t)$ is increased by 1 , and a portion $x$ of the past weights is forgotten. For ease of bookkeeping, the weights of unordered pairs are defined as symmetric weights of ordered pairs, so the weights $W(j, i, t), W(k, i, t)$ and $W(k, j, t)$ are increased as well. We write $W(e, t)$ for $W(i, j, t)$ when $e$ is the edge (unordered set) $\{i, j\}$.

It is shown in [PS03a] that the network always breaks into small cliques, with interactions occurring only among the cliques:

Theorem 2.1 In Three's Company, with population size $n \geq 4$ and any discount rate $x \in(0,1)$, with probability 1 the population may be partitioned into subsets of sizes 3, 4 and 5, such that each member of each subset chooses each other with positive limiting frequency, and chooses members outside the subset only finitely often. Every partition into sets of sizes 3, 4 and 5 has positive probability of occurring.

Simulation data is also given there. For $N=6$, if $x=.4$ (a rather steep discount rate), the network always breaks into two cliques of size 3 , as predicted by the theorem. When $N=6$ and $x=.2$, which is still a greater discount rate than one finds in most economic models, one finds, with runs of several thousand, that no such structure emerges. Instead, all six members of the population remain well connected. This is because of the exponential time scale of the transition from stable equilibria (well-connectedness is a stable equilibrium of the non-discounted model) to trapping states (two cliques of size three is the unique trapping state of the $N=6$ discounted model). Specifically, in the last section of this paper we will prove:

Theorem 2.2 In the game Three's Company, for each $N \geq 6$ there is a $\delta>0$ and numbers $c_{N}>0$ such that in Three's Company with $N$ players and discount rate $1-x$, the probability is at least $\delta$ that each player will play with each other player beyond time $\exp \left(c_{N} x^{-1}\right)$.

\footnotetext{
${ }^{4}$ Engage in some rewarding interaction such as anti-competitive price fixing
} 


\section{Trapping in one-dimensional discounted reinforcement}

In this section we analyze a one-dimensional process in which sharp quantitative results may be obtained on the exponential rate at which the time until trapping increases with $1 / x$, where $1-x$ is the discount factor. This is in keeping with our philosophy of providing sharp results on a collection of simplified models that constitute building blocks for more complicated models. In the last section we will apply the principles gleaned from this to get bounds on the exponential rate of increase of trapping time in the Three's Company model.

Let us consider a system whose state vector varies in the interval $[0,1]$ with evolution dynamics that are symmetric around an attractor at $1 / 2$, and whose transitions from state $w$ have a profile that depends on $w$ and is scaled by $x$. In analogy with models such as Three's Company, we assume that the unscaled transitions have variance bounded from below as $w$ varies over compact sub-intervals of $(0,1)$. Thus the rules of evolution of the state vector $W$ may be given in terms of probability distributions $Q_{w}$, parametrized by $w \in[0,1]$, with bounded support, satisfying $Q_{w}(s)=Q_{1-w}(-s)$, and obeying:

$$
\mathbb{P}\left(W(n+1)-W(n) \in x \cdot S \mid \mathcal{F}_{n}\right)=Q_{W(n)}(S)
$$

on the event that $W(n)$ is in a compact subinterval $I_{x}$, with $I_{x} \uparrow(0,1)$ as $x \rightarrow 0$. We assume that the mean of $Q_{w}$ is positive on $(0,1 / 2)$ and negative on $(1 / 2,1)$, but that $Q_{w}$ has both positive and negative elements in its support and varies smoothly with $w$.

As an example, one may consider a class of two-color urn models generalizing Friedman's Urn [Fr49, Fr65] in the discounted setting. An urn begins with $R(0)$ red balls and $B(0)$ black balls. At the $n^{\text {th }}$ time step, a random number $U(n)$ of red balls and $V(n)$ black balls are added to the urn. Conditional on the past, $\mathbb{P}(U(n)=k)=u_{W(n-1)}(k)$ and $\mathbb{P}(V(n)=k)=u_{1-W(n-1)}(k)$, where $W(n):=R(n) /(R(n)+B(n))$ is the state parameter, in this case the proportion of red balls, and $u_{w}$ are probability distributions on the nonnegative integers, continuously varying in the parameter $w \in[0,1]$, satisfying

$$
\frac{\sum_{k} k u_{w}(k)}{\sum_{k} k\left(u_{w}(k)+u_{1-w}(k)\right)}>w \text { for } 0<w<\frac{1}{2} .
$$

At the end of each step, all balls are reduced in weight by a factor of $1-x$. For greater specificity, one may keep in mind an example where two balls are sampled: if they are of the same color then one ball of that color is added; if they are of different colors then one ball of each color is added.

In the non-discounted system, where the step size scales as $1 / n$ at time $n$ instead of holding constant at $x$, the system is well approximated by a diffusion with incremental variance of order $n^{-2}$ and drift $n^{-1} \mu_{w}$, with $\mu_{w}$ being the mean, $\overline{Q_{w}}$, of $Q_{w}$. Thus $(2.3)$ holds with $g(t)=t^{-1}$ and $\mu(w)=\overline{Q_{w}}$. The system must converge to the unique attracting equilibrium at $1 / 2$ [Pem90]. In the discounted case, although the state must converge to 0 or 1 , the logarithm of the expected time to come near 0 or 1 may be computed in terms of the following data.

Pick any $w \in(0,1 / 2)$. The quantity

$$
Z_{w}(\lambda):=\int \exp (-\lambda y) d Q_{w}(y)
$$

is equal to 1 at $\lambda=0$. The derivative $\left.(d / d \lambda) Z_{w}(\lambda)\right|_{\lambda=0}$ is given by $\int(-y) d Q_{w}(y)$, which is negative by the assumption that $Q_{w}$ has positive mean. On the other hand, since $Q_{w}$ gives positive probability 
to negative values, we see that as $\lambda \rightarrow \infty, Z_{w} \rightarrow \infty$, and by convexity of $Z_{w}(\cdot)$ it follows that there is a unique $\lambda_{w}>0$ for which $Z_{w}\left(\lambda_{w}\right)=1$. Define

$$
\Lambda(w):=\int_{w}^{1 / 2} \lambda_{u} d u
$$

and let $C:=\Lambda(0)$.

Theorem 3.1 Let $I_{x} \uparrow(0,1)$ as $1-x \uparrow 1$, slowly enough so that transitions outside of $[0,1]$ are never possible. Let $T_{x}$ be the expectation of the first time $n$ that $W(n) \notin I_{x}$. Then as $1-x \uparrow 1$,

$$
x \log \mathbb{E} T_{x} \rightarrow C .
$$

Remark: This is essentially a large deviation problem, so the rate $C$ is not determined by the mean and variance of $Q_{w}$ but rather by the exponential moments of $Q_{w}$. In particular, there are many processes which satisfy $(2.3)$ with the same $g$ and $\mu$, but their large deviation rates depend on the fine structure of the increment distribution through the exponential moments, as captured by $Z_{w}$ and $\lambda_{w}$. The solution of this rate problem is standard; a similar analysis may be found, for example in [DZ93, Section 5.8.2].

Proof: For one inequality, we fix any $\delta>0$. Define the quantity

$$
M_{(\delta)}(t):=\exp \left((1-\delta) x^{-1} \Lambda(W(t)) .\right.
$$

Since $Q_{w}$ varies smoothly with $w$ and has bounded support, we see that as $x \rightarrow 0$,

$$
\Lambda(W(t+1))-\Lambda(W(t))=(W(t+1)-W(t))\left(-\lambda_{W(t)}+O(x)\right) .
$$

Therefore, since the conditional distribution of $x^{-1}(W(t+1)-W(t))$ given $\mathcal{F}_{t}$ is given by $Q_{W(t)}$, we see that

$$
\begin{aligned}
\mathbb{E}\left(M_{(\delta)}(t+1) \mid \mathcal{F}_{t}\right) & =M_{(\delta)}(t) \mathbb{E}\left[\exp \left((1-\delta) x^{-1}(W(t+1)-W(t))\left(-\lambda_{W(t)}+O(x)\right)\right)\right] \\
& \rightarrow M_{(\delta)}(t) Z_{W(t)}\left((1-\delta) \lambda_{W(t)}\right)
\end{aligned}
$$

uniformly in $W(t)$ as $x \rightarrow 0$. We know that $Z_{w}(\cdot)<1$ on $\left(0, \lambda_{w}\right)$, hence we may pick $x=x(\delta)$ small enough so that

$$
M_{(\delta)}(t)^{-1} \mathbb{E}\left(M_{(\delta)}(t+1) \mid \mathcal{F}_{t}\right)<1
$$

or in other words, so that $M_{(\delta)}$ is a supermartingale.

Let $I_{x}=\left[a_{x}, 1-a_{x}\right]$. Starting with $W\left(t_{0}\right) \in(1 / 2-\delta, 1 / 2)$, and stopping at the time $\tau$ when $W(\cdot)$ exits $\left[a_{x}, 1 / 2\right]$, we have for some constant $c(\delta)$ going to zero with $\delta$,

$$
\begin{aligned}
\exp \left(x^{-1} c(\delta)\right) & \geq M_{(\delta)}\left(t_{0}\right) \\
& \geq \mathbb{E}\left(M_{(\delta)}(\tau) \mid \mathcal{F}_{t_{0}}\right) \\
& \geq \mathbb{P}\left(M_{(\delta)}(\tau)<a_{x}\right) \exp \left(x^{-1} \Lambda\left(a_{x}\right)\right)
\end{aligned}
$$

which implies that

$$
\log \mathbb{P}\left(M_{(\delta)}(\tau)<a_{x}\right)<-(1-\delta) x^{-1}(\Lambda(0)+o(1))
$$


as $x \rightarrow 0$. A completely analogous argument shows that the process started in $(1 / 2,1 / 2+\delta)$ exits $\left[1 / 2,1-a_{x}\right]$ with at most this probability as well. The trajectory of $W(\cdot)$ may be decomposed into segments that begin in $[1 / 2-\delta, 1 / 2+\delta]$ and end when $W(t)-1 / 2$ changes sign or $W(t) \notin I_{x}$. We have shown that the expected number of trajectories is at least $\exp \left(x^{-1}(1-\delta)(\Lambda(0)+o(1))\right)$ as $x \rightarrow 0$, which implies that the number of time steps until exiting $I_{x}$ is at least this great, once $W(t) \in[1 / 2-\delta, 1 / 2+\delta]$. Letting $c^{\prime}(\delta)$ denote the probability of entering this interval, we see that

$$
\mathbb{E} T_{x} \geq c^{\prime}(\delta) \exp \left(x^{-1}(1-\delta)(\Lambda(0)+o(1))\right.
$$

as $x \rightarrow 0$, and finally, sending $\delta$ to zero proves that

$$
\liminf x \log \mathbb{E} T_{x} \geq C \text {. }
$$

For the other direction, define a tilted measure on the space of trajectories $\{W(t): t=0,1,2, \ldots\}$ as follows. The equation (3.1) is replaced by

$$
\tilde{\mathbb{P}}\left(W(n+1)-W(n) \in x \cdot S \mid \mathcal{F}_{n}\right)=\tilde{Q}_{W(n)}(S)
$$

where $\delta>0$ is fixed and the Radon-Nikodym derivative is given by

$$
\frac{d \tilde{Q}_{w}}{d Q_{w}}(y)=\frac{\exp [(1+\delta)(\Lambda(w+y)-\Lambda(w))]}{\int \exp [(1+\delta)(\Lambda(w+y)-\Lambda(w))] d Q_{w}(y)} .
$$

The measure $\tilde{\mathbb{P}}$ is designed to have two properties. First, the process $\{W(t)\}$ is a supermartingale on $\left[a_{x}, 1 / 2\right]$ with respect to $\tilde{\mathbb{P}}$ for sufficiently small $x$. To see this, note that this is equivalent to $\tilde{Q}_{w}$ having negative mean, which is equivalent to

$$
\int y e^{(1+\delta)(\Lambda(w+y)-\Lambda(w))} d Q_{w}(y) \leq 0
$$

for all $w \in\left[a_{x}, 1 / 2\right]$. The quantity $\Lambda(w+y)-\Lambda(w)$ is equal to $y\left(-\lambda_{w}+o(1)\right)$ as $x \rightarrow 0$, so it suffices to show that

$$
\int y e^{-(1+\delta) y \lambda_{w}} d Q_{w}(y)<0
$$

But this follows from the fact that $Z_{w}$ is convex and increases through 1 at $\lambda_{w}$ : the derivative at $(1+\delta) \lambda_{w}$ must therefore be positive, and the derivative may be identified as

$$
-\int y e^{-(1+\delta) \lambda_{w} y} d Q_{w}(y)
$$

proving the supermartingale property.

The second property is that if $\tau$ is the exit time of $\left[a_{x}, 1 / 2\right]$, then on the $\sigma$-field $\mathcal{F}_{\tau}, d \tilde{\mathbb{P}} / d \mathbb{P}$ is at most $\exp \left((1+\delta) x^{-1} \Lambda(0)\right)$. Indeed, by its definition,

$$
\frac{d \tilde{\mathbb{P}}}{d \mathbb{P}}\left(W\left(t_{0}\right), \ldots, W(\tau)\right)=\prod_{t=t_{0}}^{\tau-1} \frac{\exp \left[x^{-1}(1+\delta)(\Lambda(W(t+1)-\Lambda(W(t)))]\right.}{\int \exp [(1+\delta)(\Lambda(W(t)+y)-\Lambda(W(t)))] d Q_{W(t)}(y)} .
$$

The denominator of each factor is at least 1 by the fact that

$$
M_{(-\delta)}(t):=\exp \left((1+\delta) x^{-1} \Lambda(W(t))\right)
$$


is a submartingale when $x(\delta)$ is small enough, which is proved by a computation exactly analogous to (3.2). The product of the numerators is simply $\exp \left((1+\delta) x^{-1} \Lambda(W(\tau))-\Lambda\left(W\left(t_{0}\right)\right)\right)$, which is at most $\exp \left((1+\delta) x^{-1} \Lambda(0)\right)$, proving the second property.

Running $\tilde{\mathbb{P}}$ on $\left[a_{x}, 1 / 2\right]$ and its reflection on $\left[1 / 2,1-a_{x}\right]$, the process $1 / 2-|1 / 2-W(t)|$ is a supermartingale with incremental variance of order $x^{-2}$. The median time for it to reach a value less than $a_{x}$ is therefore at most $O\left(x^{-2}\right)$. Comparing $\tilde{\mathbb{P}}$ and $\mathbb{P}$, we find that there is a $c$ such that from any starting data, the probability of exiting $I_{x}$ by time $c x^{-2}$ is at least $(1 / 2)(d \mathbb{P} / d \tilde{\mathbb{P}}) \geq$ $(1 / 2) \exp \left(-(1+\delta) x^{-1} \Lambda(0)\right)$. Breaking into time intervals of size $c x^{-2}$, it then follows that the mean time to exit $I_{x}$ is at most $2 c x^{-2} \exp \left(C(1+\delta) x^{-1}\right)$. As this holds for any $\delta>0$ (and constants depending on $\delta$ ), this proves that

$$
\lim \sup x \log \mathbb{E} T_{x} \leq C
$$

and finishes the proof of the theorem.

\section{Proof of Theorem 2.2}

In analogy with the one-dimensional toy model, we expect to find an exponential wait to trapping in Three's Company if the non-discounted system has an attractor outside of the limit set of absorbing states of the discounted system. Unfortunately, at this point we cannot see any way to compute the large deviation rate in multi-dimensional problems. The standard multi-dimensional analogue to Theorem 3.1 is expressed as a variational result involving minimizing a functional over all paths. We settle for proving the existence of a nonzero exponential rate in $x^{-1}$. The following result will imply Theorem 2.2.

Proposition 4.1 Let the vector-valued Markov chain $\vec{W}(t)$ satisfy

$$
\mathbb{P}(\vec{W}(t+1)-\vec{W}(t) \in x S \mid \vec{W}(t))=Q_{\vec{W}(t)}(S)
$$

with $Q_{w}$ having bounded support and varying smoothly as $w$ varies over some closed neighborhood $\mathcal{N}$ of a point $\vec{c}$. Suppose there is a strong Lypunov function $V$, meaning that $V$ is smooth and bounded with $V<0$ on $\mathcal{N}^{c}, V(\vec{c})>0$ and

$$
\int V(w+y) d Q_{w}(y)>V(w)
$$

for all $w \in \mathcal{N}$. Then there is a constant $\gamma$ such that for all $\vec{h}$ in some smaller neighborhood of $\vec{c}$,

$$
\mathbb{E}_{\vec{h}} T_{x}>\gamma \exp \left(\gamma x^{-1}\right)
$$

for sufficiently small $x$, with $T_{x}$ being the time to exit $\mathcal{N}$.

Proof: Given a non-negative parameter, $\lambda$, define $M(t)=M_{\lambda}(t)=\exp (-\lambda V(\vec{W}(t)))$. Arguing as in the first half of the proof of Theorem 3.1, we see from the bounded support hypothesis that for fixed $w \in \mathcal{N}$,

$$
M_{\lambda}(t)^{-1} \mathbb{E}\left(M_{\lambda}(t+1)-M_{\lambda}(t) \mid \mathcal{F}_{t}\right)
$$


vanishes at $\lambda=0$ and has negative derivative. By compactness of $\mathcal{N}$ and smoothness of $Q_{w}$, we may choose a $\lambda>0$ so that (4.2) is negative for all $w \in \mathcal{N}$, implying that $M(t)$ is a supermartingale up to the exit time of $\mathcal{N}$. Let $\mathcal{N}^{\prime}$ be the neighborhood $\{\vec{h} \in \mathcal{N}: V(h)>V(\vec{c}) / 2\}$. Using $T_{G}$ to denote the first time $\tau \geq 0$ that $W(\tau) \in G$, we then have, for $V(\vec{h})>V(\vec{c}) / 4$,

$$
\begin{aligned}
e^{-\lambda V(\vec{c}) / 4} & \geq \mathbb{E}_{\vec{h}}(\vec{W}(0)) \\
& \geq \mathbb{E}_{\vec{h}} T_{\mathcal{N}^{c} \cup \mathcal{N}^{\prime}} \\
& \geq \mathbb{P}_{\vec{h}}\left(T_{\mathcal{N}^{c}}<T_{\mathcal{N}^{\prime}}\right) .
\end{aligned}
$$

Breaking the time $T_{\mathcal{N} c}$ into sojourns away from $\mathcal{N}^{\prime}$ then proves the theorem with $\gamma=\lambda V(\vec{c}) / 4$.

In Three's Company, if we start with the sum of the weights equal to $3 N x^{-1}$ (that is, in stationarity), then the dynamics are described exactly by (4.1). We need only check the existence of a strong Lyapunov function. This will follow if we can identify a hyperbolic attractor for the vector field $F(\cdot)$, where $F(w)$ is the mean of $Q_{w}$. Indeed, if $F$ vanishes at a point $\vec{c}$ and $d F(\vec{c})$ has eigenvalues with negative real parts, then there is a quadratic function $V$ near $\vec{c}$ satisfying $\nabla V \cdot F>0$ which we may take as the Lyapunov function. All that remains is to identify the hyperbolic attractor for the mean motion field.

The mean motion is given by a vector field $F$ on the state space. The state space is the set of non-negative real functions $X$ on the edges summing to $3 N x^{-1}$, which we think of as embedded in the cone of non-negative functions, since $F$ extends naturally via $F(\lambda X)=F(X)$. The computations are a little more convenient when we normalize the sum of weights to be $\left(\begin{array}{c}N \\ 2\end{array}\right)$. It is also convenient to let $n=N-1$ be one less than the number of agents. The attractor on which we focus is the symmetric point $\vec{c}$ defined by $c(e)=1$ for all $e$. It is immediate to verify that $F(\vec{c})=0$. In order to verify that $\vec{c}$ is an attractor for $F$, we need to compute the differential of $F$ at $\vec{c}$. Accordingly, let $\mathbf{1}_{e}$ denote the function that is 1 on $e$ and 0 elsewhere. The derivative of $F$ in the $\mathbf{1}_{e}$ direction is computed as follows.

Let the edge weights at time $t$ be given by $1+\epsilon \mathbf{1}_{e}$. The expected number of $i$ for which $f \in U(i, t)$ is $\frac{6}{n}+O(\epsilon)$ for all $f$. By symmetry, the $O(\epsilon)$ term depends only on whether $f$ shares two, one or zero endpoints with $e$. For example, in the case $f=e$, we compute the expected number of times $e$ is reinforced as follows. Let $e=\{v, w\}$. Then

$$
\mathbb{P}(e \in U(v, t))=(n-1) \frac{1+\epsilon}{\left(\begin{array}{l}
n \\
2
\end{array}\right)+(n-1) \epsilon} .
$$

The probability of $e \in U(w, t)$ is the same. For $z \neq v, w$, the probability of $e \in U(z, t)$ is exactly $\left(\begin{array}{c}n \\ 2\end{array}\right)^{-1}$. Summing yields an expected increment in $W(e, t)$ of

$$
2(n-1) \frac{1+\epsilon}{\left(\begin{array}{l}
n \\
2
\end{array}\right)+(n-1) \epsilon}+\frac{n-1}{\left(\begin{array}{l}
n \\
2
\end{array}\right)}=\frac{6}{n}+\frac{4(n-2)}{n^{2}} \epsilon+O(\epsilon)^{2} .
$$

We write this as

$$
\frac{6}{n}\left(1+\frac{2(n-2)}{3 n} \epsilon+O\left(\epsilon^{2}\right)\right)
$$

Computing, the other two expectations in this manner, we find that the expectation for $f$ at distance $j$ from $e$ is $\frac{6}{n}\left(1+B_{j} \epsilon+O\left(\epsilon^{2}\right)\right)$, where

$$
B_{0}=\frac{2(n-2)}{3 n}
$$




$$
\begin{aligned}
& B_{1}=0 \\
& B_{2}=-\frac{4}{3 n(n-1)} .
\end{aligned}
$$

From this it follows that for any $\vec{h}$,

$$
\frac{n}{6} \mathbb{E}(W(t+1) \mid W(t)=\vec{c}+\epsilon \vec{h})=\vec{c}+M \vec{h}+O\left(|\vec{h}|^{2}\right)
$$

where $M$ is a generalized circulant matrix (symmetric under the action of edge permutation on pairs of edges) with entries $B_{0}$ on the diagonal, $B_{2}$ for disjoint edges, and zero otherwise. Since $F$ is the vector field pointing toward $\mathbb{E}(W(t+1)-W(t) \mid W(t))$, the differential of $F$ is, up to the constant multiple $\frac{6}{n}$, equal to $M-I$.

The eigenvalues of a matrix such as $M$ are particularly easy to evaluate, using the rubric of association schemes (see [Ter96, Section 2.2], which is taken from [BI84, BCN89]). All such matrices are elements of the Bose-Mesener algebra $\mathcal{M}$ which, in the case of the incidence graph for edges of the complete graph, is commutative semi-simple of dimension 3. This implies that $M$ has at most three distinct eigenvalues. These may be found by computing the action of $M$ on the three shared eigenspaces common to all elements of $\mathcal{M}$.

The null eigenspace has dimension 1: $M \vec{h}=0$ if and only if $\vec{h}=\lambda \vec{c}$. The other two eigenvalues may be gotten by choosing an edge $e$ and setting the eigenvectors equal to $a_{2} H_{2}+a_{1} H_{1}+a_{0} H_{0}$, where $H_{2}=\mathbf{1}_{e}, H_{1}$ is the sum of $\mathbf{1}_{f}$ over edges $f$ sharing one vertex with $e$, and $H_{0}$ is the sum of $\mathbf{1}_{f}$ over edges $f$ disjoint from $e$. The action of $M$ on such a sum produces another such sum, and is linear, having matrix

$$
\frac{4}{3 n(n-1)}\left(\begin{array}{ccc}
\left(\begin{array}{c}
n-1 \\
2
\end{array}\right) & 0 & -1 \\
0 & \left(\begin{array}{c}
n-2 \\
2
\end{array}\right) & -2(n-3) \\
-\left(\begin{array}{c}
n-1 \\
2
\end{array}\right) & -\left(\begin{array}{c}
n-2 \\
2
\end{array}\right) & 2 n-5
\end{array}\right)
$$

with respect to $a, b$ and $c$. The left eigenvectors of this are

$$
(1,1,1) \quad, \quad\left(\frac{n-1}{2}, \frac{n-3}{4},-1\right) \quad \text { and } \quad\left(\left(\begin{array}{c}
n-1 \\
2
\end{array}\right),-\frac{n-2}{2}, 1\right) \text {. }
$$

The corresponding eigenvalues are

$$
0, \frac{2}{3} \frac{(n+1)(n-2)}{n(n-1)} \text { and } \frac{2}{3} \frac{n-3}{n-1} .
$$

The equations of mean motion are

$$
\mathbb{E}(W(t+1)-W(t) \mid W(t)-\vec{c}+\epsilon \vec{h})=\frac{6}{n} x^{-1}(M-I) \vec{h}+O\left(|\vec{h}|^{2}\right),
$$

whence the point $\vec{c}$ is attracting for sufficiently small $x$ if and only if the real parts of all eigenvalues of $M$ are less than 1 . We have identified that this is so, and therefore $\vec{c}$ is attracting. The hypotheses of Proposition 4.1 are therefore satisfied with a quadratic Lyapunov function, and Theorem 2.2 follows from Theorem 3.1. 


\section{References}

[AI97] Anderlini, L. and Ianni, A. (1997). Learning on a torus. In: The dynamics of norms, ed. C. Bicchieri, R. Jeffrey, and B. Skyrms (Cambridge University Press, Cambridge) 87-107.

[BI84] Bannai, E. and Ito, T. (1984). Algebraic combinatorics I: association schemes. Benjamin/Cummings: Menlo Park, CA.

[BCN89] Brouwer, A., Cohen, A. and Neumaier, A. (1989). Distance-regular graphs. Springer-Verlag: Berlin.

[BW00] Barrat, A. and Weigt, M. (2000). On the properties of small-world network models. Europ. Phys. J. B 13, 547.

[Ben96] Benaïm, M. (1996). A dynamical system approach to stochastic approximations. SIAM J. Control Opt. 34437 - 472.

[BH95] Benaï, M. and Hirsch, M. (1995). Dynamics of Morse-Smale urn processes. Ergodic Theory and Dynamical Systems 151005 - 1030.

[BP03] Benjamini, I. and Pemantle, R. (2003). Probabilities for cooled Brownian motion to linger near the top of a hill, and application to a market share model. Preprint.

[BL02] Bonacich, P. and Liggett, T. (2002). Asymptotics of a matrix-valued Markov chain arising in sociology. Preprint.

[BM55] Bush, R. and Mosteller, F. (1955). Stochastic models for learning. John Wiley \& Sons: New York.

[Dav90] Davis, B. (1990). Reinforced random walk. Prob. Th. Rel. Fields 84, 203-229.

[DZ93] Dembo, A. and Zeitouni, O. (1993). Large deviations techniques and applications. Jones and Bartlett: Boston.

[EP23] Eggenberger, F. and Pólya, G. (1923). Uber die Statistik verketter vorgäge. Zeit. Angew. Math. Mech. 1, 279-289.

[El193] Ellison, G. (1993). Learning, local interaction, and coordination. Econometrica 61, 10471071.

[Fr65] Freedman, D. (1965). Bernard Friedman's urn. Ann. Math. Stat. 36956 - 970.

[Fr49] Friedman, B. (1949). A simple urn model. Comm. Pure Appl. Math. 2, 59-70.

[FK93] Fudenberg, D. and Kreps, K. (1993). Learning mixed equilibria. Games and Econ. Beh. $\mathbf{5}, 320-367$.

[IT69] Iosifescu, M. and Theodorescu, R. (1969). Random processes and learning. Springer-Verlag: New York.

[Lak81] Lakshmivarahan, S. (1981). Learning algorithms: theory and applications. Springer-Verlag: New York.

[Lim01] Limic, V. (2001). Attracting edge property for a class of reinforced random walks. Preprint. http://www.math.cornell.edu/ limic/ 
[FM96] Flache, A. and Macy, M. (1996). The weakness of strong ties: collective action failure in a highly cohesive group. J. Math. Sociol. 21, 3-28.

[MS82] Maynard Smith, J. (1982). Evolution and the theory of games. Cambridge University Press: Cambridge.

[Nor72] Norman, M. (1972). Markov processes and learning models. Academic Press: New York.

[Pem90] Pemantle, R. (1990). Nonconvergence to unstable points in urn models and stochastic approximations. Ann. Probab. $18698-712$.

[PS03a] Pemantle, R. and Skyrms, B. (2003). Network formation by reinforcement learning: the long and medium run. Preprint.

[PS03b] Pemantle, R. and Skyrms, B. (2003). Reinforcement schemes may take a long time to exhibit limiting behavior. In Preparation.

[RM51] Robbins, H. and Monro, S. (1951). A stochastic approximation method. Ann. Math. Stat. 22, 400-407.

[RE95] Roth, A. and Erev, I. (1995). Learning in extensive form games: experimental data and simple dynamic models in the intermediate term. Games and Economic Behavior 8, 164212.

[Ter96] Terwilliger, P. (1996). Algebraic Combinatorics. Lecture Notes, posted January 24, 1996.

[WS98] Watts, D. and Strogatz, S. (1998) Collective dynamics of "small-world" networks. Nature 393, 440-442. 